\title{
Health care resource utilization and costs among adult patients with hemophilia A on factor VIII prophylaxis: an administrative claims analysis
}

\author{
Stacy E Croteau, MD, MMS; Keziah Cook, PhD; Lamiya Sheikh, MS; Anita Chawla, PhD; Joshua Sammon, PharmD; \\ Paul Solari, MD; Benjamin Kim, MD, MPhil; David Hinds, MHS; and Courtney D Thornburg, MD, MS
}

\section{What is already known about this subject}

- Total health care costs for hemophilia A are substantial and driven by the cost of factor VIII (FVIII) replacement concentrates and bypassing agents, especially for those on prophylaxis and those with FVIII inhibitors.

- Well-defined criteria for identifying FVIII treatment regimen and inhibitor status from administrative claims data are needed to provide reliable cost estimates for patients with hemophilia A managed with prophylaxis.

- Criteria described in previous studies may not be reflective of current clinical and reimbursement policies and may permit inclusion of a substantial number of patients with inhibitors and/or patients on episodic treatment.

\section{What this study adds}

- This study describes a structured approach to identifying a particular subset of patients with hemophilia A (those without inhibitors who are being managed with continuous FVIII prophylaxis) from administrative claims data, which uses criteria based on expected treatment patterns and place of service according to current clinical practice and reimbursement policies in the United States.

- Contemporary analysis of annual total health care costs associated with the management of hemophilia A show that per patient costs averaged $\$ 287,055$ per year among all patients receiving FVIII concentrate (any type) regardless of regimen; mean annual total health care costs for patients on continuous prophylaxis were higher, ranging from $\$ 407,752$ to $\$ 551,645$.

\section{ABSTRACT}

BACKGROUND: Standard of care for bleed prevention in patients with severe congenital hemophilia A is continuous prophylaxis with factor VIII (FVIII), typically administered intravenously 2-3 times per week in the home setting. Nonfactor prophylaxis and gene therapy are emerging novel prophylaxis strategies for hemophilia A, and it is important to compare their health economics with that of FVIII prophylaxis. Current data on resource utilization and costs in the adult hemophilia A prophylaxis population are limited, and a structured approach to analyze annual costs in these patients using administrative claims data has not been previously reported.

OBJECTIVE: To assess health care resource utilization and costs of continuous FVIII prophylaxis in commercially insured adults with hemophilia A without inhibitors.

METHODS: Administrative claims records from beneficiaries covered by major selfinsured companies in the United States from January 1999 through March 2017 (OptumHealth Care Solutions) were queried,

\author{
Author affiliations \\ Stacy E Croteau, MD, MMS, Division of \\ Hematology-Oncology, Boston Children's \\ Hospital/Harvard Medical School, Boston, \\ MA. Keziah Cook, PhD; Lamiya Sheikh, \\ MS; and Anita Chawla, PhD, Analysis \\ Group, Menlo Park, CA. Joshua Sammon, \\ PharmD; Paul Solari, MD; Benjamin Kim, \\ MD, MPhil; and David Hinds, MHS, BioMarin \\ Pharmaceutical, Novato, CA. Courtney D \\ Thornburg, MD, MS, Division of Pediatric \\ Hematology/Oncology, Rady Children's \\ Hospital, San Diego, CA.
}

AUTHOR CORRESPONDENCE:

Courtney D Thornburg, 858.966.5811; cthornburg@rchsd.org

J Manag Care Spec Pharm 2021;27(3):316-26

Copyright $@ 2021$, Academy of Managed Care Pharmacy. All rights reserved.

and records for adult patients (aged 18-64 years) diagnosed with hemophilia A who received FVIII were extracted. Three criteria were defined to distinguish patients most likely to be managed with continuous FVIII prophylaxis from those on episodic treatment based on the frequency and timing of FVIII claims over a 12-month period of continuous enrollment: (1) having $\geq 4$ FVIII claims, (2) having $\geq 6$ FVIII claims, or (3) having no gaps $>60$ days between FVIII claims. Patients with evidence of bypassing agent use were excluded. Health care resource utilization and costs were assessed for all patients with 
any FVIII use and for patients defined as being managed with continuous FVIII prophylaxis based on each criterion.

RESULTS: The analysis included 189 patients with a diagnosis code for hemophilia A (ICD 9-CM code 286.0; ICD-10-CM code D66) from January 1999 through March 2017 who had at least 12 months of continuous enrollment and at least 1 noninpatient/nonemergency department claim for FVIII concentrate (any type) during their last 12 months of continuous enrollment (overall cohort). Within the overall cohort, 118, 94, and 61 patients met the criteria for FVIII prophylaxis based on the first, second, and third definitions, respectively. Per patient mean (SD) total health care costs for the overall cohort was $\$ 287,055(306,933)$. For patients meeting criteria 1 through 3 , per patient costs ranged from $\$ 407,752(321,036)$ to $\$ 551,645(302,841)$. FVIII concentrate accounted for over $90 \%$ of costs, with mean (SD) annual FVIII costs of $\$ 264,777(292,423)$ in the overall cohort and $\$ 384,197(303,826), \$ 433,029(313,711)$, and $\$ 531,098(297,142)$ among patients meeting the respective definitions for prophylaxis.

CONCLUSIONS: This analysis highlights the substantial economic burden associated with managing adults with hemophilia A on FVIII prophylaxis, where per patient mean total annual health care costs ranged from $\$ 407,752$ to $\$ 551,645$. Over $90 \%$ of such costs were attributable to FVIII concentrate dispensed.

Hemophilia A is a rare X-linked bleeding disorder characterized by a deficiency in clotting factor VIII protein (FVIII) that affects an estimated 1 in 5,000 live male births and has an estimated prevalence of 20,000 in the United States. ${ }^{1}$ Current treatment strategies for management of hemophilia A are associated with high health care resource utilization and costs, and despite the low prevalence of hemophilia A, the condition accounts for a sizable proportion of expenditures for payers. ${ }^{2-6}$ On-demand or episodic treatment of bleeding is associated with high morbidity and low quality of life. ${ }^{7}$ Therefore, the current U.S. standard of care for severe hemophilia A without inhibitors is lifelong continuous prophylaxis to prevent bleeding events, defined as the intent to treat for 52 weeks per year with a minimum for 45 weeks per year. ${ }^{8}$ Prophylaxis with FVIII concentrate is typically given by intravenous infusion at home 2-3 times per week. ${ }^{9}$

Studies describing the economic burden of severe hemophilia A managed with FVIII prophylaxis according to the current standard of care are needed to establish a baseline with which to assess the perceived economic value of emerging treatment options and to understand their associated burden on the health care system. Administrative claims records are a valuable source of health care resource utilization and cost data, particularly for hemophilia A, where the rarity of the condition can make it challenging to identify sufficiently large samples of patients who meet select criteria of interest.

However, definitions of FVIII prophylaxis applied across previous analyses of claims data have been varied, omitted, or defined in a manner that could capture a substantial number of patients on episodic treatment, as well as patients with inhibitors. An approach to identify individuals on prophylactic FVIII therapy from claims data while minimizing known confounds, such as patients receiving episodic FVIII therapy and patients with active inhibitors who require more intense treatment, is particularly important for hemophilia A given the substantial effect they can have on cost estimates. ${ }^{2,3,5,10}$ Separately, the introduction of extended half-life (EHL) FVIII concentrates since 2014 can also drive cost increases in the United States., ${ }^{4,1-13}$ Finally, variation in how amounts paid for drugs and medical services are observed or estimated contribute to the broad distribution of reported expenditures associated with the management of hemophilia A. Together, these factors complicate the understanding of the economic burden of severe hemophilia $\mathrm{A}$ and the perceived value associated with emerging treatment options.

The current study defines a series of increasingly stringent selection criteria for identifying adult patients with hemophilia A most likely being managed with continuous FVIII prophylaxis from administrative claims data. These criteria are defined in a manner that reflect current clinical and reimbursement practices in the United States. Health care resource utilization and costs in commercially insured adult patients with hemophilia A without inhibitors managed on continuous prophylaxis with FVIII concentrate are reported.

\section{Methods}

\section{DATA}

A retrospective administrative claims analysis was conducted using data from the OptumHealth Care Solutions Claims database, which includes health insurance claims data for more than 19 million commercially insured beneficiaries covered by 84 self-insured major U.S. companies. The database includes information on patient demographics, enrollment history, medical claims, and pharmacy claims. Data extracted for this study span the period from January 1999 through March 2017.

Within these data, use of FVIII concentrate can be reflected on either medical claims or pharmacy claims, depending on each patient's specific benefit design. Both types of claims include the payment amounts specifically 
associated with FVIII concentrate, which are inclusive of price discounts but not necessarily inclusive of rebates.

\section{SAMPLE SELECTION}

Eligible patients were males aged 18-64 years with a diagnosis of hemophilia A identified based on the International Classification of Diseases, Ninth/Tenth Revision, Clinical Modification diagnosis codes (ICD-9-CM 286.0; ICD-10-CM D66). Following the algorithm described by Lyons et al. (2018) for identifying patients with hemophilia A in administrative claims databases,$^{14}$ eligible patients were also required to have at least 1 medical or pharmacy claim for FVIII concentrate in a non-inpatient or non-emergency department setting identified based on Healthcare Common Procedure Coding System (HCPCS) codes (J7182-J7188, J7190-J7192, J7207, J7210, J2597, C9136, C9137, C9140, C9267, Q2023, or Q9975) or Generic Product Identifier codes (85-10-00-10$\mathrm{XX}, 85-10-00-15-\mathrm{XX})$.

Patients were further required to have at least $1 \mathrm{FVIII}$ claim during their most recent 12 months of continuous enrollment (overall cohort). Eligible months of enrollment were defined as (a) months in which the patient enrolled in an included insurance plan and was within the age range of 18-64 years and (b) months that occurred after or contemporaneous to the patient's first noninpatient/ nonemergency department claim for FVIII concentrate. The cohort excluded patients with evidence of active FVIII inhibitors based on a claim for 1 or more bypassing agents, specifically, activated prothrombin complex concentrates (J7198/85-10-00-20-XX) and/or recombinant factor VIIa (J7189/85 100026 XX).

The cohort did not include patients receiving emicizumab because the March 2017 data cutoff date was before the drug's initial regulatory approval. Patients with a diagnosis of HIV/AIDS (ICD-9-CM codes 042-044; ICD-10-CM codes B20-B22, B24) were also excluded to make the study population more representative of the majority of U.S. patients with hemophilia A (only $10 \%$ have a history of HIV). ${ }^{1}$ These patients may require additional care to manage their infection and have often been excluded from clinical trials of emerging therapies for hemophilia A.

\section{IDENTIFICATION CRITERIA FOR PATIENTS RECEIVING PROPHYLAXIS}

Three separate criteria for identifying patients receiving continuous FVIII prophylaxis were defined based on expected treatment patterns and place of service according to current clinical practice and reimbursement policies in the United States. ${ }^{9}$ Each criterion was defined in a manner that balances incorrectly excluding patients who are truly receiving FVIII prophylaxis with incorrectly including patients receiving episodic treatment.

The first criterion categorized all patients with hemophilia A who have at least 4 noninpatient/nonemergency FVIII concentrate claims (pharmacy or medical claims) during their last 12-month period of continuous enrollment as receiving prophylaxis to capture patients regularly filling prescriptions for FVIII concentrate on 90-day supply or shorter schedules (e.g., 60-day or 30-day). The second criterion categorized patients with at least 6 noninpatient/ nonemergency department FVIII concentrate claims during the last 12-month period of continuous enrollment as receiving prophylaxis to capture patients filling prescriptions for FVIII concentrate on a 60-day supply or shorter schedule.

Criteria 1 and 2 only use the number of FVIII claims during the 12-month period to identify potential prophylaxis patients and do not use information regarding the timing of FVIII claims or the days of supply covered by each claim. The third criterion uses the timing of FVIII claims rather than the number of claims to identify patients who are likely managed with FVIII prophylaxis. Specifically, it identifies patients with no gaps of over 60 days between prescription fills for FVIII concentrate during the last 12 month period of continuous enrollment to capture patients receiving a regular supply of FVIII concentrate for prophylaxis.

To identify gaps in days of FVIII concentrate supply for criterion 3, days of supply data were used for FVIII concentrate pharmacy claims. Medical claims for FVIII concentrate do not report days of drug supply and were assumed to cover a 30-day supply. The first gap was defined as the time between the first day of the last 12-month period of continuous enrollment and the first noninpatient/nonemergency department FVIII concentrate claim in the last 12 -month period. The last gap was the time between the last noninpatient/nonemergency department FVIII concentrate claim and the end of the last 12-month period of continuous enrollment. Other gaps were defined by the time between each noninpatient/nonemergency department FVIII concentrate claim within the last 12-month period.

\section{STUDY OUTCOMES}

Health care resource utilization and cost outcomes from the most recent 12 months of continuous enrollment were reported to capture the most current management for severe hemophilia A. Health care resource utilization outcomes included the frequency of medical and pharmacy claims by place of service, FVIII concentrate claims (excludes bypassing agents and nonfactor concentrates), inpatient admissions, emergency department visits, outpatient visits, and home health visits. Health care cost outcomes included 
total medical and pharmacy costs (including those unrelated to hemophilia), medical costs by place of service, FVIII concentrate costs (which can be included on either medical or pharmacy claims), inpatient hospital costs, and emergency department costs. Comparisons between the overall cohort and prophylaxis subgroups were conducted to understand the effect of prophylaxis on health care resource utilization and costs. All costs were reported in 2017 U.S. dollars and adjusted for medical cost inflation. ${ }^{15}$

A sensitivity analysis excluded patients in the top $5 \%$ of FVIII concentrate-related costs from cost calculations. This analysis aimed to exclude potential patients with exceptionally high FVIII concentrate use, including those who have inhibitors being managed without bypassing agents. This sensitivity exclusion was based on the assumption that such patients would incur exceptionally high costs due to their need for frequent and/or high-dose infusions of FVIII concentrate and that these patients were not representative of the typical hemophilia A prophylaxis population. Exclusion of the top 5\% as a proxy for excluding patients with inhibitors was reasonable, since the study period was before the introduction of emicizumab. The exclusion was applied to the overall cohort and to each of the subgroups defined by the prophylaxis criteria.

Additional analyses of the overall cohort and the prophylaxis subgroups were conducted to compare (a) 2 age groups, the 8-26 years age group representing patients who remained eligible for coverage under parental health plans based on age limits defined in the U.S. Patient Protection and Affordable Care Act and the 27-64 years age group to capture remaining commercially insured patients, and (b) 2 time frames of the previous 12 months of continuous enrollment, 1999-2012 versus 2013-2017. The comparison of the 2 time frames aimed to account for changes to hemophilia management that may have been affected by the 2013 publication of the updated World Federation of Hemophilia guidelines, ${ }^{9}$ the introduction of new therapies since 2013, and increased prescription of prophylaxis based on results of the Joint Outcomes Study. ${ }^{9,16,17}$

\section{STATISTICAL ANALYSES}

Descriptive statistics were computed for patient characteristics and each health care resource and cost outcome. Patient characteristics included age, insurance plan type, beneficiary status, year at last 12 months eligibility start date, and region of insurance coverage. Resource utilization and costs were summarized as annual frequency of events and annual costs per patient. Descriptive statistics for resource utilization outcomes included means and SDs.
Descriptive statistics for costs included means, SDs, medians, and quartiles. One-sample location tests with finite population corrections were conducted to compare mean costs for each subgroup with the mean cost of the overall cohort. Statistical testing of differences in resource utilization were not conducted.

Comparisons of cost across age and enrollment year for the overall cohort and for each prophylaxis subgroup were conducted using parametric and nonparametric tests. Parametric chi-square tests for categorical variables and t-tests for continuous variables were used to compare patient characteristics and resource utilization. Nonparametric Wilcoxon rank sum tests were used to compare cost outcomes.

\section{Results}

\section{OVERALL COHORT AND SUBGROUP CHARACTERISTICS}

A total of 4,333 patients with a diagnosis of hemophilia A were identified from the database for January 1999 through March 2017 (Figure 1). The overall cohort included 189 patients with at least 12 months of continuous enrollment and at least 1 noninpatient/nonemergency department FVIII concentrate claim during their last 12 months of continuous enrollment. In the overall cohort, 118 patients had $\geq 4$ claims (subgroup 1); 94 patients had $\geq 6$ claims (subgroup 2); and 61 patients had no supply gaps $>60$ days (subgroup 3 ).

The median age among the overall cohort, as well as each of the subgroups, was 25.0 years (Table 1). Patients in the overall cohort were insured for a mean (SD) of 55.9 (42.6) months, with $49.7 \%$ being the primary beneficiary and $49.7 \%$ being a secondary beneficiary; distribution of mean months of insurance coverage and beneficiary status were similar across the 3 prophylaxis subgroups.

\section{HEALTH CARE COSTS}

Overall Cohort and Prophylaxis Subgroups. FVIII concentrate costs were the primary cost driver, accounting for $92.2 \%$ of total health care costs in the overall cohort, and 94.0\%-96.2\% across the 3 prophylaxis subgroups defined according to the different criteria. Per patient mean (SD) total annual health care costs among the prophylaxis subgroups were $\$ 407,752(321,036)$ for subgroup 1, $\$ 460,576$ $(331,971)$ for subgroup 2 , and $\$ 551,645(302,841)$ for subgroup 3 (Table 2). Costs incurred from home health visits and outpatient visits (both inclusive of the cost of FVIII concentrate) were the 2 largest drivers of costs reported in medical claims (Supplementary Table 1, available in online article). Mean (SD) per patient total annual health care costs 


\section{FIGURE 1 Sample Selection Flowchart}

\section{Step 1: Diagnosis}

Patients with $\geq 1$ claim for hemophilia A (ICD-9-CM: 286.0; ICD-10-CM: D66) from January 1999 through March 2017 $\mathrm{N}=4,333$

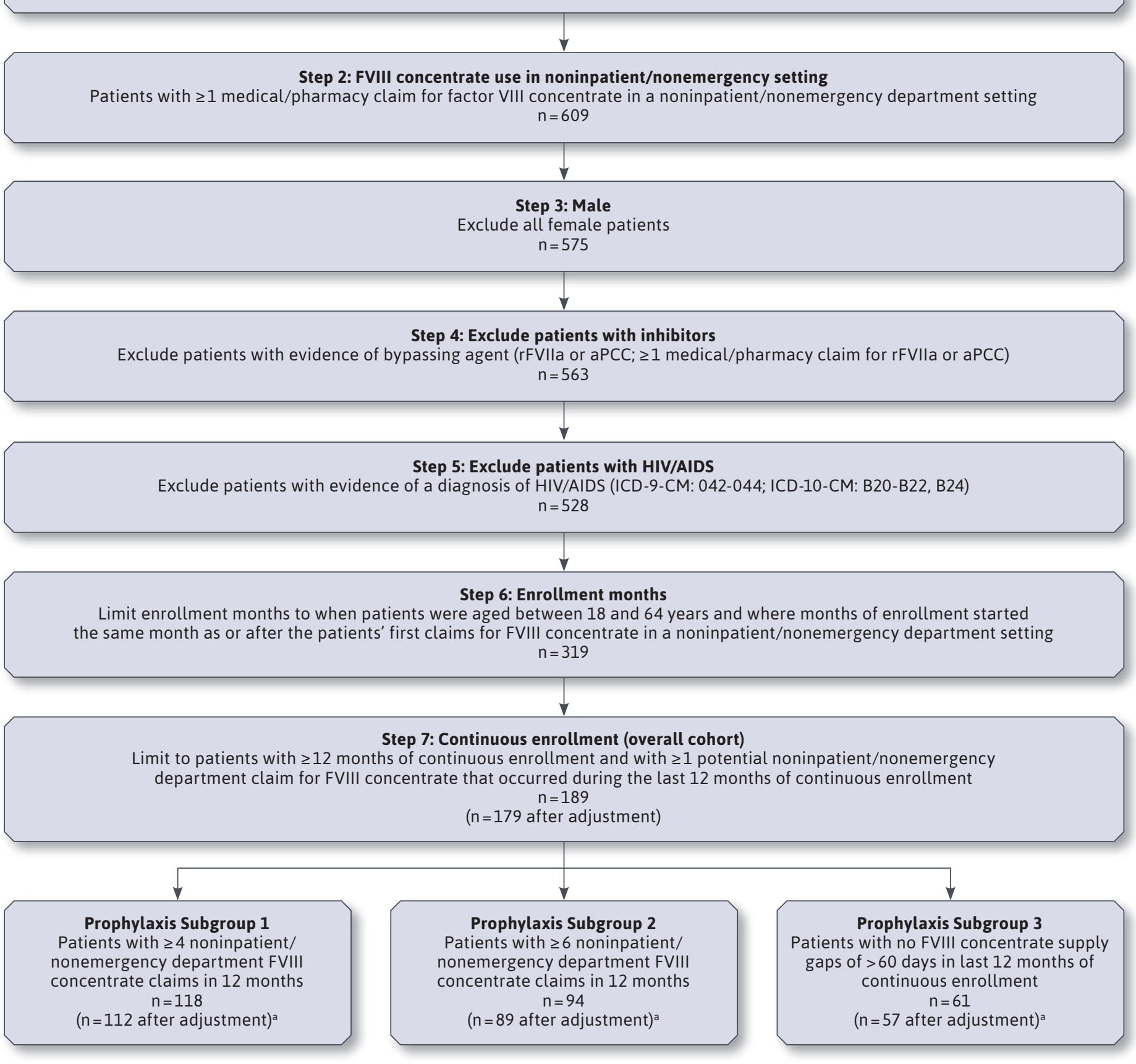

aSample sizes after adjustment are based on exclusion of patients in the top $5 \%$ of FVIII-related costs.

AIDS=acquired immune deficiency syndrome; $\mathrm{APCC}=$ activated prothrombin complex concentrate; FVIIa=activated recombinant factor VII; FVIII = factor VIII; ICD-9/10-CM = International Classification of Diseases, Ninth/Tenth Revision, Clinical Modification. 


\section{TABLE 1 Patient Characteristics}

\begin{tabular}{|c|c|c|c|c|}
\hline Characteristic & $\begin{array}{l}\text { Overall Cohorta } \\
\qquad(\mathrm{N}=189)\end{array}$ & $\begin{array}{l}\text { Prophylaxis } \\
\text { Subgroup } 1 \\
\geq 4 \text { Claims } \\
(n=118)\end{array}$ & $\begin{array}{c}\text { Prophylaxis } \\
\text { Subgroup } 2 \\
\geq 6 \text { Claims }(n=94)\end{array}$ & $\begin{array}{c}\text { Prophylaxis } \\
\text { Subgroup } 3 \\
\text { No Gaps }>60 \text { Days } \\
(n=61)\end{array}$ \\
\hline Age $^{b}$ (years), mean (SD) & $30.5 \quad(12.5)$ & $29.0 \quad(11.2)$ & $28.9 \quad(11.2)$ & $(9.5)$ \\
\hline Median (IQR) & $25.0(22-35)$ & $25.0(22-33)$ & $25.0(21-33)$ & $25.0(21-31)$ \\
\hline Average months with insurance coverage, mean (SD) & $(0.0)$ & - & - & - \\
\hline \multicolumn{5}{|l|}{ Beneficiary status, n (\%) } \\
\hline Primary beneficiary & $94 \quad(49.7)$ & $62 \quad(52.5)$ & $(53.2)$ & $(52.5)$ \\
\hline Secondary beneficiary & $94 \quad(49.7)$ & $56 \quad(47.5)$ & $44 \quad(46.8)$ & $(47.5)$ \\
\hline Unknown & $(0.5)$ & $(0.0)$ & $(0.0)$ & $(0.0)$ \\
\hline \multicolumn{5}{|l|}{ Year at last 12 months eligibility start date, n (\%) } \\
\hline $1999-2012$ & $96 \quad(50.8)$ & $68 \quad(57.6)$ & $53 \quad(56.4)$ & $(52.5)$ \\
\hline 2013-2016 & $93 \quad(49.2)$ & $50 \quad(42.4)$ & $41 \quad(43.6)$ & $(47.5)$ \\
\hline \multicolumn{5}{|l|}{ Region at last month of coverage, $n(\%)$} \\
\hline South & $68 \quad(36.0)$ & $41 \quad(34.7)$ & $30 \quad(31.9)$ & $(34.4)$ \\
\hline Midwest & $48 \quad(25.4)$ & $28 \quad(23.7)$ & $23 \quad(24.5)$ & $(26.2)$ \\
\hline Northeast & $39 \quad(20.6)$ & $27 \quad(22.9)$ & $22 \quad(23.4)$ & (23.0) \\
\hline West & $31 \quad(16.4)$ & $20 \quad(16.9)$ & $17 \quad(18.1)$ & $(14.8)$ \\
\hline Unknown & $3 \quad(1.6)$ & $(1.7)$ & $(2.1)$ & $(1.6)$ \\
\hline \multicolumn{5}{|l|}{ Insurance type at last month of coverage, $\mathbf{n}(\%)$} \\
\hline PPO & $130 \quad(68.8)$ & $85 \quad(72.0)$ & $67 \quad(71.3)$ & $39 \quad(63.9)$ \\
\hline $\mathrm{HMO}$ & $15 \quad(7.9)$ & $6 \quad(5.1)$ & $(5.3)$ & $(6.6)$ \\
\hline Indemnity & $(6.3)$ & $(5.9)$ & $(5.3)$ & $(6.6)$ \\
\hline Point of service & $26 \quad(13.8)$ & $17 \quad(14.4)$ & $14 \quad(14.9)$ & $11 \quad(18.0)$ \\
\hline Other/unknown & $6 \quad(3.2)$ & $(2.5)$ & $(3.2)$ & $(4.9)$ \\
\hline
\end{tabular}

apatients in the overall cohort may be included in more than 1 prophylaxis subgroup.

${ }^{b} \mathrm{Age}$ for the last 12 months of overall cohort is defined as age at the first month of most recent 12 months of continuous enrollment.

$H M O=$ health maintenance organization; $I Q R=$ interquartile range; $P P O=$ preferred provider organization; $S D=$ standard deviation .

for the overall cohort were significantly lower at $\$ 287,055$ $(\$ 306,933)$ compared with each prophylaxis subgroup (for all, $\mathrm{P}<0.0001)$, likely reflecting the inclusion of patients not managed with FVIII prophylaxis.

When claims for FVIII concentrate in the inpatient/ emergency department settings and patients with the top $5 \%$ of FVIII concentrate costs were excluded from the overall cohort, per patient mean (SD) total health care costs in the overall cohort decreased by $25.5 \%$ to $\$ 213,941$ (\$211,503). The percentage changes in the prophylaxis subgroups decreased by $13.6 \%-17.0 \%$ to equal $\$ 338,274(235,834)$ for subgroup 1, $\$ 385,548(248,087)$ for subgroup 2, and $\$ 476,545$ $(221,334)$ for subgroup 3.
Age Group and Enrollment Time Period. Health care costs across select categories also varied according to age group and enrollment time period (Supplementary Table 2, available in online article). In the overall cohort, median FVIII concentrate costs for patients in the 18-26 years age group were significantly higher than for patients in the 27-64 years age group ( $\$ 213,902$ vs. $\$ 110,057, P=0.04$ ). Patients whose last 12 months of continuous enrollment were between 2013 and 2017 had significantly higher median pharmacy costs but significantly lower medical costs than those whose last 12 months of continuous enrollment fell between 1999 and 2012 (pharmacy: $\$ 102,441$ vs. $\$ 27,235, P=0.02$; medical: $\$ 2,054$ vs. $\$ 22,981, P=<0.0001)$. 


\section{TABLE 2 Per Patient Health Care Costs During Last 12 Months of Continuous Enrollment}

\begin{tabular}{|c|c|c|c|c|c|c|c|c|}
\hline \multirow[b]{2}{*}{ Costs Category } & \multicolumn{2}{|c|}{$\begin{array}{l}\text { Overall Cohort }{ }^{\text {a }} \\
(\mathrm{N}=189)\end{array}$} & \multicolumn{2}{|c|}{$\begin{array}{c}\text { Prophylaxis Subgroup } 1 \\
\geq 4 \text { Claims } \\
(\mathrm{n}=118)\end{array}$} & \multicolumn{2}{|c|}{$\begin{array}{c}\text { Prophylaxis Subgroup } 2 \\
\geq 6 \text { Claims } \\
\text { ( } n=94)\end{array}$} & \multicolumn{2}{|c|}{$\begin{array}{c}\text { Prophylaxis Subgroup } 3 \\
\text { No Gaps }>60 \text { Days } \\
(n=61)\end{array}$} \\
\hline & Mean (SD) & Median (IQR) & Mean (SD) & Median (IQR) & Mean (SD) & Median (IQR) & Mean (SD) & Median (IQR) \\
\hline Total costs & $\begin{array}{l}287,055 \\
(306,933)\end{array}$ & $\begin{array}{l}182,980 \\
(49,418- \\
414,261)\end{array}$ & $\begin{array}{l}407,752^{b} \\
(321,036)\end{array}$ & $\begin{array}{l}351,065 \\
(182,236- \\
555,242) \\
\end{array}$ & $\begin{array}{l}460,576^{\mathrm{b}} \\
(331,971)\end{array}$ & $\begin{array}{c}394,353 \\
(227,628- \\
589,754)\end{array}$ & $\begin{array}{l}551,645^{b} \\
(302,841)\end{array}$ & $\begin{array}{c}444,974 \\
(356,310- \\
654,298)\end{array}$ \\
\hline Medical expenditures & $\begin{array}{c}90,305 \\
(185,007)\end{array}$ & $\begin{array}{c}5,104 \\
(531-66,407) \\
\end{array}$ & $\begin{array}{l}121,368^{c} \\
(218,986)\end{array}$ & $\begin{array}{c}8,862 \\
(563-116,815) \\
\end{array}$ & $\begin{array}{l}138,175^{b} \\
(234,214)\end{array}$ & $\begin{array}{c}11,101 \\
(825-169,075)\end{array}$ & $\begin{array}{l}134,076^{\mathrm{b}} \\
(229,581)\end{array}$ & $\begin{array}{c}4,852 \\
(762-144,665) \\
\end{array}$ \\
\hline Pharmacy expenditures & $\begin{array}{l}196,750 \\
(282,718)\end{array}$ & $\begin{array}{l}60,738 \\
(1,694- \\
317,869)\end{array}$ & $\begin{array}{l}286,384^{\mathrm{b}} \\
(318,098)\end{array}$ & $\begin{array}{l}208,315 \\
(5,610- \\
429,029)\end{array}$ & $\begin{array}{l}322,401^{b} \\
(339,706)\end{array}$ & $\begin{array}{c}245,148 \\
(5,610- \\
457,503)\end{array}$ & $\begin{array}{l}417,569^{b} \\
(358,089)\end{array}$ & $\begin{array}{c}376,894 \\
(162,360- \\
547,919)\end{array}$ \\
\hline $\begin{array}{l}\text { FVIII concentrate costs } \\
\text { (medical and pharmacy) }\end{array}$ & $\begin{array}{l}264,777 \\
(292,423)\end{array}$ & $\begin{array}{l}168,676 \\
(34,526- \\
392,502)\end{array}$ & $\begin{array}{l}384,197^{b} \\
(303,826)\end{array}$ & $\begin{array}{c}314,684 \\
(168,676- \\
537,904)\end{array}$ & $\begin{array}{l}433,029^{\mathrm{b}} \\
(313,711)\end{array}$ & $\begin{array}{c}375,586 \\
(218,517- \\
576,314)\end{array}$ & $\begin{array}{l}531,098^{\mathrm{b}} \\
(297,142)\end{array}$ & $\begin{array}{l}434,663 \\
(349,707- \\
646,245)\end{array}$ \\
\hline $\begin{array}{l}\text { FVIII concentrate costs } \\
\text { (medical and pharmacy, } \\
\text { excluding IP and ED) }\end{array}$ & $\begin{array}{c}261,989 \\
(292,842)\end{array}$ & $\begin{array}{l}162,516 \\
(33,697- \\
380,810)\end{array}$ & $\begin{array}{l}383,389^{b} \\
(304,421)\end{array}$ & $\begin{array}{c}314,684 \\
(168,676- \\
537,904)\end{array}$ & $\begin{array}{l}432,136^{b} \\
(314,439)\end{array}$ & $\begin{array}{c}375,042 \\
(210,959- \\
576,314)\end{array}$ & $\begin{array}{l}529,864^{\mathrm{b}} \\
(298,627)\end{array}$ & $\begin{array}{c}434,663 \\
(349,707- \\
646,245) \\
\end{array}$ \\
\hline $\begin{array}{l}\text { FVIII concentrate } \\
\text { pharmacy costs }\end{array}$ & $\begin{array}{l}193,875 \\
(283,739)\end{array}$ & $\begin{array}{c}60,082 \\
(0-287,341)\end{array}$ & $\begin{array}{l}282,759^{b} \\
(320,096)\end{array}$ & $\begin{array}{c}207,629 \\
(0-428,072)\end{array}$ & $\begin{array}{l}318,411^{b} \\
(342,173)\end{array}$ & $\begin{array}{c}245,148 \\
(0-455,493)\end{array}$ & $\begin{array}{l}413,233^{b} \\
(362,599)\end{array}$ & $\begin{array}{c}376,746 \\
(135,378- \\
569,629) \\
\end{array}$ \\
\hline $\begin{array}{l}\text { FVIII concentrate } \\
\text { medical costs }\end{array}$ & $\begin{array}{c}68,113 \\
(173,486)\end{array}$ & $\begin{array}{c}0 \\
(0-27,600)\end{array}$ & $\begin{array}{l}100,630^{c} \\
(212,228)\end{array}$ & $\begin{array}{c}0 \\
(0-64,913)\end{array}$ & $\begin{array}{l}113,724^{b} \\
(227,518)\end{array}$ & $\begin{array}{c}0 \\
(0-71,558)\end{array}$ & $\begin{array}{l}116,631^{b} \\
(225,654)\end{array}$ & $\begin{array}{c}0 \\
(0-64,913)\end{array}$ \\
\hline
\end{tabular}

Notes: Costs are in 2017 U.S. dollars. P values based on 1-sample location tests with finite population corrections comparing mean costs between overall cohort and individual prophylaxis subgroups. Median costs were not compared.

apatients in the overall cohort may be included in more than 1 prophylaxis subgroup.

${ }^{b} P<0.0001$ vs. overall cohort.

${ }^{c} P<0.0005$ vs. overall cohort.

$E D=$ emergency department; $F V I I I=$ factor $V I I I ; I P=$ inpatient; $I Q R=$ interquartile range; $S D=$ standard deviation .

\section{HEALTH CARE RESOURCE UTILIZATION}

Overall Cohort and Prophylaxis Subgroups. Aside from FVIII concentrate, the most commonly used health care resources were outpatient visits, followed by home health agency visits, and emergency department visits (Table 3). In the prophylaxis subgroups, the proportion of patients with at least 1 inpatient visit ranged from $17.8 \%$ in subgroup 1 to $21.3 \%$ in subgroup 3, whereas the percentage of patients with at least 1 outpatient visit ranged from $89.8 \%$ in subgroup 1 to $95.1 \%$ in subgroup 3. Across the prophylaxis subgroups, $70.3 \%-78.7 \%$ of patients had at least 1 pharmacy claim, and $29.5 \%-36.4 \%$ had at least 1 medical claim for FVIII concentrate. In the overall cohort, $18.5 \%$ of patients had at least 1 inpatient visit, and $90.5 \%$ of patients had at least 1 outpatient visit. On claims for FVIII concentrate, $67.2 \%$ of patients had at least 1 pharmacy claim, and $38.6 \%$ had at least 1 medical claim.

Age Group and Enrollment Time Period. Health care resource utilization was generally similar across the age groups for 18-26 years and 27-64 years of the overall cohort, except for mean outpatient utilization, which was higher in the older age group $(P=0.005)$. Patients whose last 12 months of continuous enrollment began between 2013 and 2017 on average had fewer claims for home health visits and FVIII concentrate than patients whose last 12 months enrollment began between 1999 and 2012 ( $P=0.01$ for home health visits; $\mathrm{P}=0.04$ for FVIII concentrate claims).

\section{Discussion}

The current study provides updated health care resource utilization and cost estimates using administrative claims data for U.S. patients with hemophilia A without inhibitors who use FVIII concentrates according to prophylaxis regimens. This study illustrates the importance of identifying patients who are likely on FVIII prophylaxis when using claims data to assess resource utilization and costs. The described approach and criteria provide an initial 


\section{TABLE 3 Health Care Resource Utilization During Last 12 Months of Continuous Enrollment}

\begin{tabular}{|c|c|c|c|c|c|c|c|c|}
\hline Health Care Resource Category & \multicolumn{2}{|c|}{$\begin{array}{l}\text { Overall Cohort }{ }^{\mathrm{a}} \\
\qquad(\mathrm{N}=189)\end{array}$} & \multicolumn{2}{|c|}{$\begin{array}{c}\text { Prophylaxis Subgroup } 1 \\
\geq 4 \text { Claims } \\
(n=118)\end{array}$} & \multicolumn{2}{|c|}{$\begin{array}{c}\text { Prophylaxis Subgroup } 2 \\
\geq 6 \text { Claims } \\
(n=94)\end{array}$} & \multicolumn{2}{|c|}{$\begin{array}{l}\text { Prophylaxis Subgroup } 3 \\
\text { No Gaps }>60 \text { Days } \\
(n=61)\end{array}$} \\
\hline \multicolumn{9}{|c|}{ Proportion with evidence of utilization, $n$ (\%) } \\
\hline Inpatient admission & 35 & $(18.5)$ & 21 & $(17.8)$ & 19 & $(20.2)$ & 13 & $(21.3)$ \\
\hline Emergency department visit & 56 & $(29.6)$ & 36 & $(30.5)$ & 28 & $(29.8)$ & 18 & $(29.5)$ \\
\hline Outpatient visit & 171 & $(90.5)$ & 106 & $(89.8)$ & 87 & $(92.6)$ & 58 & $(95.1)$ \\
\hline Home health visit & 63 & $(33.3)$ & 36 & $(30.5)$ & 31 & $(33.0)$ & 17 & $(27.9)$ \\
\hline Other visit & 27 & $(14.3)$ & 17 & $(14.4)$ & 14 & $(14.9)$ & 12 & $(19.7)$ \\
\hline \multicolumn{9}{|l|}{ FVIII concentrate claims } \\
\hline Pharmacy claim & 127 & $(67.2)$ & 83 & $(70.3)$ & 67 & $(71.3)$ & 48 & $(78.7)$ \\
\hline Medical claim & 73 & $(38.6)$ & 43 & $(36.4)$ & 35 & $(37.2)$ & 18 & $(29.5)$ \\
\hline \multicolumn{9}{|l|}{ Per patient utilization, mean (SD) } \\
\hline Inpatient admission & 0.3 & $(0.6)$ & 0.3 & $(0.6)$ & 0.3 & $(0.7)$ & 0.2 & $(0.5)$ \\
\hline Emergency department visit & 0.7 & $(2.0)$ & 0.7 & $(2.1)$ & 0.7 & $(2.2)$ & 0.8 & $(2.6)$ \\
\hline Outpatient visit & 8.1 & $(12.5)$ & 8.0 & $(13.1)$ & 8.9 & $(14.3)$ & 8.6 & $(11.1)$ \\
\hline Home health visit & 2.4 & $(6.5)$ & 3.2 & (7.7) & 3.8 & $(8.5)$ & 3.1 & $(6.8)$ \\
\hline Other visit & 0.2 & $(0.8)$ & 0.3 & $(0.9)$ & 0.3 & (1.0) & 0.4 & $(1.2)$ \\
\hline FVIII concentrate claims & 7.8 & $(9.5)$ & 11.3 & $(10.5)$ & 13.1 & (11.1) & 14.4 & (11.8) \\
\hline $\begin{array}{l}\text { FVIII concentrate claims } \\
\text { (noninpatient/nonemergency) }\end{array}$ & 7.6 & (9.4) & 11.2 & $(10.4)$ & 12.9 & $(11.0)$ & 14.2 & $(11.7)$ \\
\hline
\end{tabular}

apatients in the overall cohort may be included in more than 1 prophylaxis subgroup.

FVIII= factor VIII; SD = standard deviation

framework that could support future efforts to standardize the criteria for identifying patients managed with FVIII prophylaxis and, thus, facilitate comparability across studies while mitigating potential confounders.

Annual health care costs in the prophylaxis subgroups were significantly higher compared with the overall cohort. Moreover, estimates of annual total health care costs and FVIII concentrate costs in the overall cohort were generally consistent with estimates from past literature..$^{2,5,18}$ Notably, the overall cohorts described in these previous studies similarly included patients managed with prophylaxis, as well as those managed with episodic treatment.

Annual costs estimates for FVIII prophylaxis have previously been reported in a pair of studies that analyzed patient records in the American Thrombosis and Hemostasis Network dataset (ATHNdataset). ${ }^{4,5}$ The ATHNdataset study reported higher estimated annual projected costs for FVIII prophylaxis based on prescribed clotting factor use, at $\$ 690,144$ for patients receiving standard half-life FVIII concentrate and $\$ 753,480$ for those receiving EHL FVIII concentrate. ${ }^{4}$ Differences in cost estimates likely relate to differences in data granularity, as well as the cost benchmarks used. The ATHNdataset contains treatment regimen data allowing for more accurate identification of patients on FVIII prophylaxis, but the dataset lacks specific cost data. Estimated annual costs of FVIII prophylaxis reported in the study were based on use and median wholesale acquisition cost, which does not reflect the confidential discount rates and rebates negotiated between drug manufacturers and payers (discounts are reflected in the current claims analysis). Notably, the ATHNdataset study highlights how the projected decrease in use of clotting factor concentrate with EHL products (in units/person per year) does not result in stable or reduced cost of care due to their higher per unit costs. The earlier cost analysis study reporting estimated median annual direct costs of FVIII prophylaxis at $\$ 286,198$ sourced clotting factor therapy cost information from average sales prices, which take into account most confidential discount rates and rebates. ${ }^{5}$ However, the study time period (pre-2013) does not capture current clinical practice and reimbursement policies. 
Analysis of the 2 age groups within the overall cohort showed patients in the 18-26 years age group had higher FVIII concentrate costs than those in the 27-64 years age group, suggesting higher rates of FVIII prophylaxis use in younger patients who may have started on prophylaxis during childhood. In the analysis of time periods, the lower costs of FVIII concentrate in medical claims in the later time period (2013-2017) were offset by increases in FVIII concentrate costs in pharmacy claims, consistent with the shift in payment model for FVIII concentrate from a medical to pharmacy benefit.

Excluding patients with the top 5\% of FVIII concentrate costs led to double-digit percentage decreases in mean total health care costs. As patients with inhibitors tend to have much higher health care costs than patients without inhibitors, ${ }^{3}$ costs reported from this sensitivity analysis may provide a more representative approximation of the true costs of managing patients without inhibitors receiving FVIII prophylaxis.

Subgroup 3 used the most stringent criteria for FVIII prophylaxis, and the per patient mean total costs after excluding high-cost outliers (as well as FVIII concentrate costs from inpatient/emergency department settings) decreased by $13.6 \%$, from $\$ 551,645$ to $\$ 476,545$. The latter figure provides a conservative estimate of the mean annual costs for managing patients who could be candidates for emerging treatments for using administrative claims data, including gene therapy-based modalities.

\section{LIMITATIONS}

Several limitations should be considered when interpreting the results of this analysis. Administrative claims data lack details associated with clinical or laboratory data that would provide information on timing of actual administration of FVIII concentrate relative to time of dispensing, the prescribed dose, use of FVIII for continuous or intermittent prophylaxis, documented use of prophylaxis, clinical and laboratory confirmation of disease severity and presence of inhibitors, and indication of home health visits.

Although the inclusion criteria for each prophylaxis subgroup were defined in a manner to progressively exclude more patients who could be receiving episodic treatment rather than prophylaxis, patients receiving episodic treatment could have been included into 1 or more prophylaxis subgroups if the frequency and timing of their claims for FVIII concentrate were similar to that of patients on FVIII prophylaxis.

Conversely, patients receiving prophylaxis may also have been incorrectly excluded under each criteria. Criterion 1 would correctly categorize patients regularly filling prescriptions for FVIII concentrate on a 90-day supply or shorter schedules (e.g., 60-day or 30-day) as receiving prophylaxis. However, it would have also incorrectly included patients who experience sufficient breakthrough bleeds to require restocking FVIII 4 or more times during a year. Fewer patients receiving episodic treatment would have been incorrectly included under criterion 2, but patients on a 90-day supply schedule would have been incorrectly excluded. Under criterion 3, all prophylaxis patients with only FVIII pharmacy claims with complete days of supply data or with FVIII medical claims covering 30 days or fewer of FVIII concentrate would be correctly categorized. Although some patients receiving episodic treatment with 4 or more evenly spaced FVIII claims would be miscategorized as receiving prophylaxis under criterion 3 , the requirement for even spacing would result in fewer miscategorized patients than under criteria 1 or 2 .

Criterion 3 may also have excluded patients managed with FVIII prophylaxis who received a 90 day supply of FVIII concentrate just before their last 12 months of continuous enrollment, although such patients are expected to be in the minority and could still be included in subgroup 1. Of note, drug supply gap was used for criterion 3 , since information on days drug supply were not consistently available in claims for FVIII concentrate and could not be reliably inferred due to the lack of patient weight information in claims data.

Patients with inhibitors may have also been present in the study sample despite having defined criteria for their exclusion, in particular, those who had achieved partial tolerance and used higher than typical doses of FVIII concentrate without bypassing agents. During sample selection, positive inhibitor status was inferred based on a claims record for bypassing agents.

Separately, although drug costs reported in claims data can capture negotiated discounts between drug manufactures and payers, they may lack information on manufacturer rebates, which would result in reporting of drug costs higher than that actually incurred by payers. Although the introduction of EHL FVIII concentrates has increased FVIII concentrate-associated costs, the full magnitude of this effect may not be well captured in the current dataset due to the March 2017 data cutoff date. The cutoff date was also before the introduction of emicizumab, a bispecific monoclonal antibody, which is now approved in the United States for prophylaxis in patients with hemophilia A, with and without inhibitors.

Finally, the reported costs may not have been representative of beneficiaries enrolled in public health plans such as Medicaid or in capitated health plans such as health maintenance organizations. Analysis of larger administrative claims databases could facilitate refinement to the 
described algorithm and associated criteria for identifying patients likely to be managed with FVIII prophylaxis by enabling examination of trends in dose and days of drug supplied across a larger sample and over more recent time periods.

\section{Conclusions}

The current study describes a novel approach for examining costs and health care resource utilization in claims data for patients with hemophilia A without inhibitors who are likely being managed with continuous FVIII prophylaxis. Mean total annual health care costs incurred by these patients ranged from $\$ 407,752$ for subgroup 1 to $\$ 551,645$ for subgroup 3, with FVIII concentrate driving more than $90 \%$ of overall health care costs. These data provide updated cost estimates for managing patients with continuous FVIII prophylaxis that reflect the current standard of care and may support future studies to understand the health economics of emerging treatments for hemophilia A, including gene therapy-based modalities. The study highlights the effect of how the definitions used will influence the estimates observed, particularly for conditions such as hemophilia A. Analysis of larger administrative claims databases could facilitate future refinements to the described approach and associated criteria for identifying patients likely to be managed with FVIII prophylaxis.

\section{DISCLOSURES}

This study was funded by BioMarin Pharmaceutical, which was involved in protocol development, analysis plan development, data interpretation, manuscript preparation, and publication decisions. All authors contributed to protocol development, analysis plan development, data interpretation, and manuscript development. All authors maintained control over the final content. Sammon, Solari, Kim, and Hinds are employees and shareholders of BioMarin Pharmaceutical. Cook, Sheikh, and Chawla are employees of Analysis Group, a consulting company that was contracted by BioMarin Pharmaceutical to conduct this study and develop the manuscript. Croteau has received professional fees from BioMarin Pharmaceutical, Bayer, CSL Behring, Genentech, and Pfizer. Thornberg has received professional fees from BioMarin Pharmaceutical, Genentech, Novo Nordisk, Sanofi, and Spark Therapeutics, as well as research funding from Novo Nordisk and Sanofi.

\section{ACKNOWLEDGMENTS}

Medical writing assistance was provided by Kai-Jye Lou, an employee of Analysis Group, and Jacob Fang, a former employee of Analysis Group.

\section{REFERENCES}

1. Centers for Disease Control and Prevention. Community counts: factor VIII and factor IX. 2020. Accessed January 26, 2021. https://www.cdc.gov/ncbddd/ hemophilia/communitycounts/datareports/2020-3/table-2-factor.html

2. Shrestha A, Eldar-Lissai A, Hou N, Lakdawalla DN, Batt K. Real-world resource use and costs of haemophilia A-related bleeding. Haemophilia. 2017;23(4):e267-e275.

3. Armstrong EP, Malone DC, Krishnan S, Wessler MJ. Costs and utilization of hemophilia A and B patients with and without inhibitors. J Med Econ. 2014;17(11):798-802.

4. Croteau SE, Cheng D, Cohen AJ, et al. Regional variation and cost implications of prescribed extended half-life factor concentrates among U.S. Haemophilia Treatment Centres for patients with moderate and severe haemophilia. Haemophilia. 2019;25(4):668-75.

5. Zhou ZY, Koerper MA, Johnson KA, et al. Burden of illness: direct and indirect costs among persons with hemophilia A in the United States. J Med Econ. 2015;18(6):457-65.
6. O'Hara J, Hughes D, Camp C, Burke T, Carroll L, Diego DG. The cost of severe haemophilia in Europe: the CHESS study. Orphanet J Rare Dis. 2017;12(1):106.

7. Nugent D, O'Mahony B, Dolan G. Value of prophylaxis vs on-demand treatment: application of a value framework in hemophilia. Haemophilia. 2018;24(5):755-65.

8. Blanchette VS, Key NS, Ljung LR, Manco-Johnson MJ, van den Berg HM, Srivastava A. Definitions in hemophilia: communication from the SSC of the ISTH. J Thromb Haemost. 2014;12(11):1935-39.

9. Srivastava A, Brewer AK, Mauser-Bunschoten EP, et al. Guidelines for the management of hemophilia. Haemophilia. 2013;19(1):e1-e47.

10. Rocha P, Carvalho M, Lopes M, Araujo F. Costs and utilization of treatment in patients with hemophilia. BMC Health Serv Res. 2015;15:484.

11. Bowen K, Gleason P. Incremental cost of switching to extended half-life coagulation factor products to treat hemophilia among 15 million commercially insured members. Paper presented at: AMCP Annual Meeting; April 23-26, 2018; Boston, MA.

12. Chhabra A, Fogarty PF, Tortella BJ, et al. Real-world analysis of dispensed international units of coagulation factor VIII and resultant expenditures for hemophilia A patients: a comparison between standard half-life and extended half-life products. Manag Care. 2018;27(10):39-50.

13. Spurden D, Chhabra A, Tortella B, et al. Real world data analysis of US claims database on coagulation factor VIII expenditures in patients with Hemophilia A: Standard Half-life versus extended half-life products. Paper presented at: 64th Annual Meeting of the Scientific Standardization Committee of the International Society on Thrombosis and Haemostasis; 18-21 July, 2018; Dublin, Ireland.

14. Lyons J, Desai V, Xu Y, et al. Development and validation of an algorithm for identifying patients with hemophilia A in an administrative claims database. Value Health. 2018;21(9):1098-103. 
15. U.S. Bureau of Labor Statistics.

Consumer Price Index (CPI) databases. All urban consumers, U.S. medical care 2017. 2019. Accessed January 26, 2021. https:// www.bls.gov/cpi/data.htm

16. Jackson MD, O'Brien SH, Stanek J, Dunn AL, Kerlin BA. Inpatient health care utilization in children with hemophilia before and after the joint outcome study publication. J Pediatr Hematol Oncol.

2019;41(5):e284-e289.
17. Manco-Johnson MJ, Abshire TC, Shapiro AD, et al. Prophylaxis versus episodic treatment to prevent joint disease in boys with severe hemophilia. N Engl J Med. 2007;357(6):535-44.
18. Guh S, Grosse SD, McAlister S, Kessler CM, Soucie JM. Healthcare expenditures for males with haemophilia and employersponsored insurance in the United States, 2008. Haemophilia. 2012;18(2):268-75. 